\title{
High-mass X-ray binaries in a cosmological context
}

\author{
María Celeste Artale ${ }^{1,2, *}$, Leonardo J. Pellizza ${ }^{1,2}$, \\ Patricia B. Tissera ${ }^{1,2}$ \& I. Felix Mirabel ${ }^{1,3}$ \\ ${ }^{1}$ Instituto de Astronomía y Física del Espacio, CONICET-UBA, CC 67 Suc 28, Ciudad \\ Autónoma de Buenos Aires (1428), Argentina \\ ${ }^{2}$ Consejo Nacional de Investigaciones Científicas y Técnicas, Argentina \\ ${ }^{3}$ CEA-Saclay, IRFU/DSM/Service d'Astrophysique, 91191, Gif-sur-Yvette, France \\ *email: mcartale@iafe.uba.ar
}

\begin{abstract}
Recent observational and theoretical results suggest that the production rates and luminosities of high-mass X-ray binaries depend on metallicity. To test this prediction, we combine HMXB population synthesis results with numerical simulations of galaxy formation to produce synthetic populations of HMXBs in star-forming galaxies, and compare the model predictions to observations of HMXB populations in nearby and high-redshift galaxies. Our models show a fair agreement with observations only when the HMXB production and luminosities are assumed to depend strongly on metallicity.
\end{abstract}

Keywords. X-ray: binaries, galaxies - stars: evolution - galaxies: abundances, evolution

\section{Introduction}

High-mass X-ray binaries (HMXBs) are systems composed of a compact object (neutron star NS or black hole BH) and a massive companion star (Fabbiano 2006). Recent studies suggest that these sources, especially those in which the compact object is a $\mathrm{BH}$, might play a fundamental role as sources of energy feedback into the interstellar medium, particularly at the early stages of the Universe (Mirabel et al. 2011). This is supported by current theoretical models, which predict that the HMXB production rate and possibly luminosity increase at low metallicities (Belczynski et al. 2004; Georgy et al. 2009; Linden et al. 2010). Testing these predictions against observations is needed to shed light on the aforementioned problem. We present here a novel scheme to model HMXB population properties, which couples population synthesis results to galaxy catalogs from hydrodynamical cosmological simulations of structure formation, allowing a fair comparison with observations. We use this scheme to investigate the possible chemical dependence of the HMXB formation rate and luminosity.

\section{Cosmological Simulations, Population Synthesis and Results}

We produce synthetic populations of HMXBs using galaxy catalogs from hydrodynamic cosmological simulations made with a version of GADGET-3 (Scannapieco et al. 2006), consistent with the concordance $\Lambda$-CDM model, which include star formation, metaldependent cooling, chemical enrichment, multiphase treatment of the gas, and supernovae feedback (SNII, SNIa). The simulations describe a periodical box of $10 h^{-1} \mathrm{Mpc}$ side; the matter within it is described initially by $2 \times 230^{3}$ particles of gas and dark matter. Initial abundances of the gas are $X=0.76, Y=0.24$, and the chemical enrichment of baryons is followed by computing the abundances of twelve major elements. The cosmological parameters of the simulation are $\Omega_{\Lambda}=0.7, \Omega_{\mathrm{m}}=0.3, \Omega_{\mathrm{b}}=0.04, \sigma_{8}=0.9$ and $H_{0}=$ 
$100 h \mathrm{~km} \mathrm{~s}^{-1} \mathrm{Mpc}^{-1}$, where $h=0.7$. A friends-of-friends technique was applied to select virialized structures and the SUBFIND algorithm to select substructures within them. These simulations have been applied to the study of the Tully-Fisher relation, obtaining a good agreement with observations (for further details see De Rossi et al. 2010).

We investigate the chemical dependences of the progenitor of HMXBs in nearby and distant galaxies by combining the results of population synthesis models with the galaxy catalogs from the simulations. Considering the short lifetimes of HMXBs, we take from the galaxy catalogs all stellar populations with lifetimes below $100 \mathrm{Myr}$ as potential progenitor of these sources. We selected this value to avoid small-number statistical effects. We estimate the number of NS and BH in binary systems for each stellar population following the models of Georgy et al. (2009) and Belczynski et al. (2004). The total Xray luminosity of a galaxy is computed by collecting the contributions of HMXBs, which are estimated by using a power law X-ray luminosity function (XLF Mineo et al. 2012). A possible chemical dependence of the XLF is taken into account by varying the mean HMXB luminosity in different models. To account for the fact that the exact lifetime and duty cycle of HMXBs can not be accurately modeled, we include a normalization factor constant in time and the same for all galaxies, which is left as a free parameter to be constrained by observations. The model predictions are compared to the data of two HMXB samples: one composed of nearby galaxies (Mineo et al. 2012) and the other of high-redshift objects (Cowie et al. 2012). To compare our models with observed HMXB populations, we take into account the detectability of the X-ray emission of each galaxy and the selection effects present in both samples. Our model reproduces fairly well the observed trend relating the X-ray luminosities of star-forming nearby galaxies to their star formation rates. However, the dispersion of this relation reproduced only by models in which the HMXB production depends on metallicity. For high-redshift galaxies, the observed evolution of the mean X-ray luminosity of star-forming galaxies with redshift agrees only with those models with a strong chemical dependent XLF. Hence, our results suggest that both the number of HMXBs in a population and their luminosity increase with metallicity, as models predict (Belczynski et al. 2004; Linden et al. 2010).

\section{Acknowledgments}

MCA would like to thank the IAU for its financial support to attend this meeting. MCA, LJP, and PBT acknowledge support from the European Commission's Framework Programme 7, through the Marie Curie International Research Staff Exchange Scheme LACEGAL (PIRSES-GA-2010-269264).

\section{References}

Belczynski, K., Sadowski, A., \& Rasio, F. A. 2004, ApJ, 611, 1068

Cowie, L. L., Barger, A. J., \& Hasinger, G. 2012, ApJ, 748, 50

De Rossi, M. E. \& Tissera, P. B. 2010, A\&A 519,89

Fabbiano, G. 2006, ARA\&A, 44, 323

Fragos, T., Lehmer, B., Tremmel, M., et al. 2012, arXiv:1206.2395

Georgy, C., Meynet, G., Walder, R., Folini, D., \& Maeder, A. 2009, A\&A, 502, 611

Kaaret, P., Schmitt, J., \& Gorski, M. 2011, ApJ, 741, 10

Linden, T., Kalogera, V., Sepinsky, J. F., et al. 2010, ApJ, 725, 1984

Mirabel, I. F., Dijkstra, M., Laurent, P., Loeb, A., \& Pritchard, J. R. 2011, A\&AA, 528, 149

Mineo, S., Gilfanov, M., \& Sunyaev, R. 2012, MNRAS, 419, 2095

Scannapieco, C., Tissera, P. B., White, S. D. M., \& Springel, V. 2006, MNRAS, 371, 1125 